\title{
Propiedades tecnológicas de la fibra de Alpacas Huacaya blanca (Vicugna pacos) en la Comunidad de Chapimarca, Apurímac - Perú
}

\author{
Technological properties of the fibre of white Huacaya alpacas (Vicugna pacos) in \\ the Community of Chapimarca, Apurimac - Peru \\ Virgilio Machaca Machaca ${ }^{1,3.5}$, Fany Callonza Pizarro ${ }^{1}$, Valeriano Paucara Ocsa ${ }^{1}$, \\ Víctor A. Bustinza Choque ${ }^{3}$, Jesús E. Quispe Coaquira ${ }^{2,3}$, Roger Machaca Machaca ${ }^{4}$, \\ Víctor R. Cano Fuentes ${ }^{1}$, Katherine D. Arias Huamani ${ }^{1}$
}

\section{Resumen}

En el sector de Queulla, comunidad de Chapimarca, provincia de Grau, Región Apurímac, se determinaron las propiedades textiles de la fibra de alpacas Huacaya blancas, en función a factores sexo, región corporal y grupo etario. Los análisis de la fibra se realizaron en el Laboratorio de Fibras y Lanas de la Universidad Nacional de Huancavelica. Las alpacas juveniles presentaron un menor diámetro de fibra (MDF), mayor factor de confort (FC), mayor finura al hilado (SF) y mejor índice de curvatura (IC) que las adultas $(\mathrm{p}<0.05)$. Los machos presentaron un mejor FC y finura al filado (SF) que las hembras $(\mathrm{p}<0.05)$. En general la fibra de la zona del costillar y de la grupa fueron de similar calidad, pero superior a la fibra de la paleta $(\mathrm{p}<0.05)$, Se concluye que la fibra producida en la zona del estudio se ubica dentro de las fibras de buena calidad.

Palabras clave: fibra natural, morfología, factor, altoandina, Huacaya

\footnotetext{
${ }^{1}$ Facultad de Medicina Veterinaria y Zootecnia, Universidad Nacional Micaela Bastidas de Apurímac, Abancay, Perú

${ }^{2}$ Facultad de Medicina Veterinaria y Zootecnia Universidad Nacional del Altiplano, Puno, Perú

${ }^{3}$ Instituto de Investigación y Promoción de Camélidos Sudamericanos, de la UNA Puno, Perú

${ }^{4}$ Instituto Superior Tecnológico Público de Sangarará Cusco, Perú

${ }^{5}$ E-mail:machacav@hotmail.com
}

Recibido: 21 de octubre de 2020

Aceptado para publicación: 15 de mayo de 2021

Publicado: 24 de agosto de 2021

CLos autores. Este artículo es publicado por la Rev Inv Vet Perú de la Facultad de Medicina Veterinaria, Universidad Nacional Mayor de San Marcos. Este es un artículo de acceso abierto, distribuido bajo los términos de la licencia Creative Commons Atribución 4.0 Internacional (CC BY 4.0) [https:// creativecommons.org/licenses/by/4.0/deed.es] que permite el uso, distribución y reproducción en cualquier medio, siempre que la obra original sea debidamente citada de su fuente original 
In the Queulla sector, community of Chapimarca, province of Grau, Apurímac Region, the textile properties of the fibre of white Huacaya alpacas were determined, according to factors sex, body region and age group. The fibre analyses were carried out in the Fibre and Wool Laboratory of the National University of Huancavelica. The juvenile alpacas had a smaller fibre diameter (MDF), a higher comfort factor (FC), a higher yarn fineness (SF) and a better curvature index (IC) than the adult ones $(\mathrm{p}<0.05)$. Males presented better FC and yarn fineness $(\mathrm{SF})$ than females $(\mathrm{p}<0.05)$. In general, the fibre of the rib area and the rump were of similar quality, but superior to the fibre of the shoulder $(p<0.05)$. It is concluded that the fibre produced in the study is within the fibres of good quality.

Key words: natural fibre, morphology, factor, high Andean, Huacaya

\section{INTRODUCCIÓN}

En la región andina, la crianza de camélidos constituye una actividad muy importante, ya que para el hombre que habita entre 4000 a 5000 msnm es casi la única fuente de ingresos económicos. La población de alpacas en el Perú es de 3590000 y la Región Apurímac ocupa el quinto lugar con 219113 cabezas (CENAGRO, 2012). La alpaca es fundamentalmente productora de fibra de excelentes cualidades textiles y de gran demanda nacional e internacional (Bustinza, 2001; Matute et al., 2009; Quispe et al., 2016).

La cantidad y calidad de la producción de fibra de alpaca están en función de factores genéticos y ambientales. Entre los primeros resaltan el sexo, edad, raza (Bustinza, 2001; Quispe et al., 2016) y color de vellón (Bustinza, 2001; McGregor y Butler, 2004; Renieri et al., 2007), y entre los factores ambientales se encuentra la alimentación, ubicación geográfica y clima (Franco et al., 2009; Quispe, 2019). La producción de fibra depende fundamentalmente del funcionamiento de los folículos pilosos, lo cual determina respuestas en la longitud, diámetro y resistencia a la tracción de las fibras. Por ejemplo, se encuentran diferencias mor- fológicas notables entre alpacas Huacaya y Suri; asimismo, el diámetro de la fibra engrosa con la edad (Bustinza et al., 1985, Bustinza, 2001; Wuliji et al., 2000; McGregor y Butler, 2004). De otra parte, el crecimiento de la fibra es sensible a la calidad de la alimentación (Bustinza, 2001; Franco y San Martín, 2007; Franco et al., 2009). De la misma manera, la campaña alpaquera y la estacionalidad de los factores climáticos proporcionan distintas respuestas en la velocidad de crecimiento del vellón y sus características (Bustinza, 2001, Quispe, 2019).

Se argumenta que, en el país, la calidad de la fibra de alpaca ha decrecido notoriamente, trayendo consigo un deterioro progresivo en el precio y en el ingreso de los criadores altoandinos, que la producción anual de fibra extrafina oscila entre 7 y $12 \%$ del total de la producción, y que la fina representa el $22 \%$ y la semi-fina el $46 \%$; es decir, la fibra ha engrosado y se ha tornado seca y quebradiza (De los Ríos, 2006). Empero, en el mercado, han surgido nuevos atributos como el factor de comodidad y la finura al hilado (Baxter y Cottle, 2010; Wood, 2003), así como la asignación de valor al rizo que está vinculado con el índice de curvatura de la fibra (McGregor, 2014). De otro lado, la demanda de prendas confeccionadas con fibras finas $y$ altamente confortables ha ido en aumento 
(McColl et al., 2006). A nivel de la Región Apurímac solo se disponen de los estudios de Vásquez et al. (2015), Machaca et al. (2017) y Ramos (2018) sobre las características tecnológicas de la fibra de alpaca.

En la Región Apurímac, las alpacas pertenecen a pequeños productores agrupados en comunidades campesinas, bajo un sistema tradicional e incipiente desarrollo, y la fibra la comercializan a través de una extensa red de intermediarios. En este entorno subsiste un injusto sistema de asignación de precios, bajo supuestos de que la fibra de alpaca proveniente de las comunidades no reúne los requerimientos de la industria textil (Quispe et al., 2016). Empero, la industria textil considera a la fibra de alpaca como una fibra especial y las prendas que se confeccionan con ellas están clasificadas como artículos de lujo (Wang et al., 2002).

En tal contexto, el presente estudio tuvo como objetivo determinar las propiedades tecnológicas (Diámetro medio de la fibra, Desviación estándar del diámetro de fibra, Coeficiente de variación del diámetro de fibra, Factor de confort, Finura de hilado e Índice de curvatura) en función al sexo, región corporal y categoría etaria de la fibra de alpacas de un área de la Región Apurímac.

\section{Materiales y Métodos}

\section{Lugar de Estudio}

La investigación se realizó en el sector de Queulla, comunidad de Chapimarca, distrito de Chuquibambilla, provincia de Grau, Región de Apurímac, Perú, ubicada en la cordillera seca, a una altitud de $4647 \mathrm{msnm}$. La zona tiene un clima seco y frío, con dos estaciones definidas (estiaje, de abril a octubre, y lluvias, de noviembre a marzo). En época de lluvias, entre enero y marzo, las praderas brindan abundante biomasa vegetal, a diferencia del resto del año. Las alpacas se crían bajo un sistema de pastoreo extensivo, sin recibir suplementación alimenticia alguna.

\section{Material Biológico}

Se tomaron muestras de tres zonas del cuerpo (paleta, costillar y grupa) de 120 alpacas Huacaya clínicamente sanas, de color blanco, clasificadas por edad (juveniles y adultos) y sexo (hembras y machos). El muestreo se realizó en el mes de noviembre en horas de la mañana mediante un corte con una tijera curva. Las muestras de fibra (5 g) se colocaron en bolsitas de polietileno y etiquetadas con los datos pertinentes.

\section{Análisis de Laboratorio}

Las muestras se analizaron con un equipo OFDA 2000 (Optical Fibre Diameter Analysis) (Qi et al., 1994; Marler et al., 2002; Wood, 2003), perteneciente al Laboratorio de Fibras y Lanas de la Universidad Nacional de Huancavelica. El laboratorio estuvo acondicionado a una temperatura promedio de $18{ }^{\circ} \mathrm{C}$ y humedad relativa de $65 \%$, según el reglamento de la International Wool Textile Organisation (IWTO-47, 2007).

\section{Análisis Estadístico}

Se analizaron los siguientes atributos de la fibra: Diámetro de fibra (MDF), desviación estándar del diámetro de fibra (SDMFD), Coeficiente de variabilidad (CVFD), Factor de confort (CF), Índice de curvatura (FC) y Finura al hilado (SF). Los datos se analizaron utilizando la aplicación R v. 3.0.2 (R Core Team, 2014).

\section{Resultados y Discusión}

\section{Diámetro Medio y Factor de Confort}

Los estadísticos del MFD y del CF de la fibra según los factores sexo, grupo etario y región corporal se presentan en el Cuadro 1. Los promedios generales de $22.10 \pm 3.13$ $\mu \mathrm{m}$ para MFD y $92.74 \pm 8.68 \%$ para $\mathrm{CF}$ 
Cuadro 1. Diámetro de fibra (MDF) y factor de confort (FC) de la fibra de alpaca del Sector de Q'ueulla - Chapimarca, Apurímac, según sexo, edad y región corporal

\begin{tabular}{lccc}
\hline Variables & $\mathrm{n}$ & $\begin{array}{c}\mathrm{MDF} \pm \mathrm{DE} \\
(\mu \mathrm{m})\end{array}$ & $\begin{array}{c}\mathrm{FC} \pm \mathrm{DE} \\
(\%)\end{array}$ \\
\hline $\begin{array}{l}\text { Sexo } \\
\text { Hembra }\end{array}$ & 180 & $22.41 \pm 3.12^{\mathrm{a}}$ & $91.70 \pm 9.62^{\mathrm{a}}$ \\
Macho & 180 & $21.78 \pm 3.12^{\mathrm{a}}$ & $93.78 \pm 7.52^{\mathrm{b}}$ \\
Edad & & & \\
$\quad$ Juvenil & 177 & $20.09 \pm 2.14^{\mathrm{a}}$ & $96.93 \pm 3.56^{\mathrm{a}}$ \\
$\quad$ Adulto & 183 & $24.05 \pm 2.67^{\mathrm{b}}$ & $88.70 \pm 10.15^{\mathrm{b}}$ \\
Región corporal & & & \\
$\quad$ Paleta & 120 & $22.74 \pm 3.33^{\mathrm{a}}$ & $90.52 \pm 11.33^{\mathrm{b}}$ \\
$\quad$ Costillar & 120 & $21.61 \pm 2.93^{\mathrm{b}}$ & $94.10 \pm 6.77^{\mathrm{a}}$ \\
$\quad$ Grupa & 120 & $21.94 \pm 3.04^{\mathrm{ab}}$ & $93.61 \pm 6.76^{\mathrm{a}}$ \\
\hline Media general & 360 & $22.10 \pm 3.13$ & $92.74 \pm 8.68$ \\
\hline ab. Superíndices diferentes indican diferencia significativa $(\mathrm{p}<0.05)$ &
\end{tabular}

muestran niveles de categoría excelente para la fibra de alpaca estudiada.

La fibra de los machos tuvo un mayor y significativo FC sobre las hembras $(\mathrm{p}<0.05)$. Las alpacas juveniles presentaron valores superiores para MFD y FC que los adultos $(\mathrm{p}<0.05)$. Con relación a la región corporal, las zonas del costillar y grupa presentaron una mejor MDF y FC que la zona de la paleta $(\mathrm{p}<0.05$; Cuadro 1).

Similares resultados para el MFD fueron hallados por Machaca et al. (2017) y Ramos (2018) en rebaños de la Región Apurímac; en tanto mejores finuras de fibra de alpaca fueron reportados por Ormachea et al. (2015), Vásquez et al. (2015) y Barrionuevo (2019) y Llactahuamani et al. (2020). De otro lado, fibras más engrosadas fueron halladas por Castillo y Zacarías (2014); Roque y Ormachea (2018); Meza (2018) en la sierra peruana, y por Lupton et al. (2006) en Estados Unidos de América y McGregor y Butler (2004) en Australia.

El sexo no tuvo un efecto de importancia en el MFD, lo que corrobora reportes de varios investigadores (Castillo y Zacarías, 2014; Ormachea et al., 2015; Roque y Ormachea, 2018; Ramos, 2018; Barrionuevo, 2019), aunque otros señalan diferencias significativas en el efecto del factor sexo (Vásquez et al., 2015; Machaca et al., 2017; Meza, 2018).

Los resultados permiten señalar que la edad guarda relación con la finura de la fibra; es decir, las fibras de animales más jóvenes exhiben mayor finura (Bustinza, 2001; Machaca et al., 2017; Meza, 2018; Ramos 2018; Barrionuevo, 2019). La diferencia del MDF entre alpacas juveniles y adultas es de aproximadamente $4 \mu \mathrm{m}$, lo cual es de im- 
Cuadro 2. Desviación estándar del diámetro de fibra - SD(FD) y coeficiente de variabilidad - CV(FD) de la fibra de alpaca del Sector de Q'ueulla Chapimarca, Apurímac, según sexo, edad y región corporal

\begin{tabular}{lccc}
\hline Variables & $\mathrm{n}$ & $\begin{array}{c}\mathrm{SD}(\mathrm{FD}) \pm \mathrm{DE} \\
(\mu \mathrm{m})\end{array}$ & $\begin{array}{c}\mathrm{CV}(\mathrm{FD}) \pm \mathrm{DE} \\
(\%)\end{array}$ \\
\hline $\begin{array}{l}\text { Sexo } \\
\text { Hembra }\end{array}$ & 180 & $4.68 \pm 1.14^{\mathrm{a}}$ & $19.14 \pm 3.24^{\mathrm{a}}$ \\
$\quad$ Macho & 180 & $4.32 \pm 0.97^{\mathrm{b}}$ & $18.95 \pm 2.70^{\mathrm{a}}$ \\
Edad & & & \\
$\quad$ Juvenil & 177 & $4.12 \pm 0.82^{\mathrm{a}}$ & $19.62 \pm 2.64^{\mathrm{a}}$ \\
$\quad$ Adulto & 183 & $4.87 \pm 1.16^{\mathrm{b}}$ & $18.49 \pm 3.18^{\mathrm{b}}$ \\
Región corporal & & & \\
$\quad$ Paleta & 120 & $4.70 \pm 1.11^{\mathrm{a}}$ & $19.26 \pm 3.09^{\mathrm{a}}$ \\
$\quad$ Costillar & 120 & $4.37 \pm 1.08^{\mathrm{b}}$ & $18.95 \pm 2.98^{\mathrm{ab}}$ \\
$\quad$ Grupa & 120 & $4.42 \pm 1.00^{\mathrm{ab}}$ & $18.93 \pm 2.88^{\mathrm{b}}$ \\
\hline Media general & 360 & $4.50 \pm 1.07$ & $19.05 \pm 2.98$ \\
\hline
\end{tabular}

portancia a nivel comercial. En virtud de esto, para propósitos de producción de fibra de alta calidad es conveniente mantener solamente animales jóvenes en el rebaño o intensificar la selección a favor de la finura a través de serios programas de mejora genética (Quispe et al., 2009a).

Respecto al FC, Ormachea et al. (2015), Vásquez et al. (2015) y Barrionuevo (2019) hallaron valores superiores al presente estudio, en tanto que valores similares fueron reportados por otros autores (Roque y Ormachea, 2018; Arizaca, 2018; Ramos, 2018), así como valores inferiores (Meza, 2018; Gil, 2017; Castillo y Zacarías, 2014; Machaca et al., 2017). En términos generales, el FC está directamente relacionado con la finura de la fibra, pues a mayor finura, la fibra brindará mayor comodidad para atender a las exigencias del consumidor de la prenda de vestir.
Con base al MFD y el CF, la fibra producida en la Comunidad de Chapimarca, sector de Queulla, del distrito de Chuquibambilla, de la provincia Grau de Apurímac, es de buena calidad, ya que se encuentra dentro de los requerimientos exigidos por la industria textil que pide 22.00 de MFD y $95 \%$ de fibras con diámetro menor a $30 \mu \mathrm{m}$ (McGregor, 2006; Matute et al., 2009; Botha and Hunter, 2010; Quispe et al., 2016).

\section{Desviación Estándar del Diámetro de Fibra - SD(FD) y Coeficiente de Variabi- lidad - CV(FD)}

Los estadísticos del SD(FD) y del $\mathrm{CV}$ (FD) de la fibra según los factores sexo, grupo etario y región corporal se presentan en el Cuadro 2.

La SD(DF) de las hembras fue menor $(p<0.05)$ que el de los machos. Lo cual se encuentra vinculado a la función reproductiva 
de las hembras (Lupton et al., 2006; Quispe et al., 2009b y Quispe et al., 2021)). Las alpacas juveniles presentaron mejores valores para $\mathrm{SD}(\mathrm{FD})$ y $\mathrm{CV}(\mathrm{FD})$ que los adultos $(p<0.05)$. Con relación a la región corporal, las zonas del costillar y grupa presentaron una mejor MDF y FC que la zona de la paleta $(p<0.05$; Cuadro 1). Esto indica que la fibra de las alpacas juveniles muestra menor variación en la muestra, pero mayor variación entre vellones (Botha y Hunter, 2010).

Con relación a la región corporal, las zonas del costillar y grupa presentaron una mejor MDF y FC que la zona de la paleta $(p<0.05$; Cuadro 1). Por el factor región corporal, las $\mathrm{SD}(\mathrm{FD})$ y $\operatorname{los} \mathrm{CV}(\mathrm{FD})$ fueron menores en la zona del costillar y grupa con relación a la zona de la paleta $(\mathrm{p}<0.05)$. Sobre el particular, Castillo y Zacarías (2014) señalan que los valores descienden desde la región del cuello hacia la parte caudal del animal. Por otro lado, los resultados del $\mathrm{CV}(\mathrm{FD})$ fueron inferiores a los reportes de otros autores (Vásquez et al., 2015; Machaca et al., 2017; Ramos, 2018; Barrionuevo, 2019), lo que presupone que la fibra de las alpacas del estudio tiene mayor homogeneidad de atributos, lo cual es relevante los propósitos de la industria textil.

Los resultados indican que no existen razones fundamentadas para señalar que la fibra de las alpacas proveniente de los rebanos comunales no sea de buena calidad, sea por su finura o confort o la variación de esta; por el contrario, y por su condición de núcleos cerrados, probablemente con algún grado de consanguinidad, producen fibras naturales que son fácilmente incorporadas en los procesos de la industria textil.

\section{Finura de Hilado (SF) e Índice de Cur- vatura (IC)}

Los estadísticos del SF e IC de la fibra según los factores sexo, grupo etario y región corporal se presentan en el Cuadro 3. El promedio general de SF fue de $21.33 \pm 3.12 \mu \mathrm{m}$ y para IC de $35.11 \pm 7.34 \% \mathrm{~mm}$.
La SF de la fibra de los machos fue significativamente menor que de las hembras $(p<0.05)$. En forma similar, las alpacas juveniles tuvieron una menor SF y un mayor IC que los adultos $(\mathrm{p}<0.05)$; es decir, una fibra de mayor calidad. Con relación a la región corporal, las zonas del costillar y grupa presentaron una mejor SF e IC que la zona de la paleta ( $<<0.05$; Cuadro 3$)$.

La importancia del IC se relaciona con el engarzado durante el proceso textil; en consecuencia, el IC de la fibra de alpacas Huacaya correspondería a la clasificación de baja curvatura de Botha y Hunter (2010) (30 $-40 \% \mathrm{~mm}$ ).

Cabe señalar que las fibras con alta curvatura tienen un menor diámetro y una alta relación con la frecuencia de rizos en la mecha; así mismo, existe una fuerte relación entre la MFD y el FC de la fibra, donde fibras con alta curvatura tienen fibras con menor diámetro (Fish et al., 1999). Asimismo Flores (2017) reporta valores superiores en alpacas de dos años $(40.87 \pm 7.09 \% / \mathrm{mm})$ que en alpacas de cuatro años $(41.85 \pm 6.93 \% \mathrm{~mm})$, aunque sin diferencias significativas; sin embargo los resultados del presente estudio fueron inferiores a los valores de $38.35^{\circ} / \mathrm{mm}$ en alpacas de dos años reportados por Roque y Ormachea (2018), posiblemente debido a la procedencia de los animales y al tamaño de la muestra.

Respecto al SF, los hallazgos del presente estudio son superiores a los reportes de Barrionuevo (2019) y Vásquez et al. (2015), pero inferiores respecto a los de Roque y Ormachea (2018), Machaca et al. (2017) y Ramos (2018). En virtud de ello, se puede señalar que la fibra producida en el espacio de estudio puede ubicarse dentro de las fibras de buena calidad.

\section{Categorización de la Fibra}

A manera de reflexión, en el país existe la Norma Técnica del Perú (NTP N. ${ }^{\circ}$ 231.301.204) que clasifica a la fibra de alpa- 
Cuadro 3. Finura al hilado (SF) e Índice de curvatura (IC) de la fibra de alpaca del Sector de Q'ueulla - Chapimarca, Apurímac, según sexo, edad y región corporal

\begin{tabular}{lccc}
\hline Variables & $\mathrm{n}$ & $\begin{array}{c}\mathrm{SF} \pm \mathrm{DE} \\
(\mu \mathrm{m})\end{array}$ & $\begin{array}{c}\mathrm{IC} \pm \mathrm{DE} \\
(\% / \mathrm{mm})\end{array}$ \\
\hline $\begin{array}{l}\text { Sexo } \\
\text { Hembra }\end{array}$ & 180 & $21.73 \pm 3.17^{\mathrm{a}}$ & $34.92 \pm 7.84^{\mathrm{a}}$ \\
$\quad$ Macho & 180 & $20.93 \pm 3.03^{\mathrm{b}}$ & $35.29 \pm 6.82^{\mathrm{a}}$ \\
Edad & & & \\
$\quad$ Juvenil & 177 & $19.41 \pm 2.15^{\mathrm{a}}$ & $37.35 \pm 7.74^{\mathrm{a}}$ \\
$\quad$ Adulto & 183 & $23.19 \pm 2.77^{\mathrm{b}}$ & $32.94 \pm 6.22^{\mathrm{b}}$ \\
Región corporal & & & \\
$\quad$ Paleta & 120 & $22.10 \pm 3.39^{\mathrm{a}}$ & $34.54 \pm 7.33^{\mathrm{a}}$ \\
$\quad$ Costillar & 120 & $20.84 \pm 2.88^{\mathrm{b}}$ & $35.52 \pm 7.52^{\mathrm{b}}$ \\
$\quad$ Grupa & 120 & $21.05 \pm 2.95^{\mathrm{b}}$ & $35.26 \pm 7.19^{\mathrm{ab}}$ \\
\hline Media general & 360 & $21.33 \pm 3.12$ & $35.11 \pm 7.34$ \\
\hline
\end{tabular}

ca en función a la finura en seis clases: $\mathrm{Su}$ per baby, Baby, Fleece, Medium fleece, Huarizo y Gruesa. Por otro lado, Wang et al. (2002) señala que para favorecer la comercialización de la fibra alpaca en Australia, se sigue a partir de 2001 una nueva práctica de clasificación, muy similar a la NTP; siendo las clases las siguientes: Superfine, Fine, Medium, Strong y Extra strong (Cuadro 4).

Con base a lo anterior, los resultados encontrados en la comunidad del estudio permiten afirmar que la fibra alpaca es de alta calidad; ya que el $26 \%$ de ella se encuentra en el nivel de Super Baby (o Superfine); $40 \%$ en el nivel de Baby (o fine), en tanto la diferencia pertenece a Fleece (24\%), Medium fleece (8\%). Empero, los dos primeros representan el $66 \%$ del total de la fibra muestreada, la cual tiene menos de $26.5 \mu \mathrm{m}$ según la NTP, y menos de $27 \mu \mathrm{m}$ en la clasi- ficación australiana. De la misma manera, Mamani (2012) trabajando con fibra de cuatro centros de acopio comunales concluye que la fibra de las alpacas Huacaya blancas de las comunidades y/o unidades pequeñas reúnen las condiciones de fibra de calidad, por su finura y alto rendimiento.

\section{Correlaciones}

En el Cuadro 5 y Figura 1 se muestran los coeficientes de correlación entre las características tecnológicas de fibra de alpacas Huacaya del estudio.

Se observa una asociación excelente entre MFD y SF $(r=0.95)$, aceptable entre $\mathrm{CF}$ y $\mathrm{SF}(-0.88)$ y entre MFD y $\mathrm{CF}(\mathrm{r}=$ $0.88)$, en tanto que habría una asociación regular entre las variables DS(MFD) y SF ( $r=$ $0.70)$. Los valores de coeficientes de correlación por debajo de $r=0.30$ señalarían la 
Cuadro 4. Clasificación de la fibra de acuerdo con la Norma Técnica del Perú (NTP $\mathrm{N}^{\mathrm{o}} 231.301 .204$ ) y la Australian classing

\begin{tabular}{|c|c|c|c|c|}
\hline \multicolumn{2}{|c|}{ NTP } & \multicolumn{2}{|c|}{ Australian Classing } & \multirow{2}{*}{$\begin{array}{l}\text { Ubicación de } \\
\text { la fibra del } \\
\text { estudio }(\%)\end{array}$} \\
\hline Grupos & $\begin{array}{c}\text { Rango de } \\
\text { finura }(\mu \mathrm{m})\end{array}$ & Grupos & $\begin{array}{c}\text { Rango de } \\
\text { finura }(\mu \mathrm{m})\end{array}$ & \\
\hline Super baby & $<20$ & Superfine & $<20$ & 26 \\
\hline Baby & $20.1-23.0$ & Fine & $20.1-23.0$ & 40 \\
\hline Fleece & $23.1-26.5$ & Medium & $23.1-27.0$ & 24 \\
\hline Medium fleece & $26.6-29.0$ & Strong & $27.1-32.0$ & 8 \\
\hline Huarizo & $29.1-31.5$ & Extra strong & $>32.0$ & 1 \\
\hline Gruesa & $>31.5$ & & & 1 \\
\hline
\end{tabular}

Cuadro 5. Correlaciones fenotípicas entre las características de la fibra de alpaca del sector de Queulla - Chapimarca, Grau, Apurímac

\begin{tabular}{|c|c|c|c|c|c|c|}
\hline & $\operatorname{MDF}(\mu \mathrm{m})$ & $\mathrm{SD}(\mathrm{FD}), \mu \mathrm{m}$ & $\mathrm{CV}(\mathrm{FD}), \%$ & $\mathrm{CF}, \%$ & $\mathrm{SF}, \mu \mathrm{m}$ & $\mathrm{IC}, \% / \mathrm{mm}$ \\
\hline $\mathrm{MDF}, \mu \mathrm{m}$ & 1.00 & & & & & \\
\hline $\mathrm{SD}(\mathrm{FD}), \mu \mathrm{m}$ & $0.53 *$ & 1.00 & & & & \\
\hline $\mathrm{CV}(\mathrm{FD}), \%$ & $-0.16^{*}$ & $0.56^{*}$ & 1.00 & & & \\
\hline $\mathrm{CF}, \%$ & $-0.84^{*}$ & $-0.63^{*}$ & -0.06 & 1.00 & & \\
\hline $\mathrm{SF}, \mu \mathrm{m}$ & $0.95 *$ & $0.70 *$ & 0.03 & $-0.88 *$ & 1.00 & \\
\hline $\mathrm{IC}, \mathrm{o} / \mathrm{mm}$ & $-0.46^{*}$ & $-0.26^{*}$ & -0.01 & $0.38 *$ & $-0.45^{*}$ & 1.00 \\
\hline
\end{tabular}

MDF: Media del diámetro de fibra, SD(FD): Desviación estándar de la media del diámetro de fibra, CV(FD): Coeficiente de variación de la media del diámetro de fibra, CF: Factor de confort, SF: Factor de hilado, IC: Índice de curvatura, $* p<0.05$

ausencia del grado de asociación entre características. En virtud de ello, existen asociaciones positivas y negativas que tendrían aplicación utilitaria en la industria textil.

Al respecto, el MDF evidencia alto grado de asociación con el FC y FH; es decir, a mayor finura no solo habrá mayor rendimiento al hilado, sino que también la fibra fina ofrecerá mayor comodidad (menor efecto de picazón). De la misma manera, la relación del DSMDF con el FH, FC y CVMDF expresarían una mayor uniformidad en el DM e hilado de la fibra. 

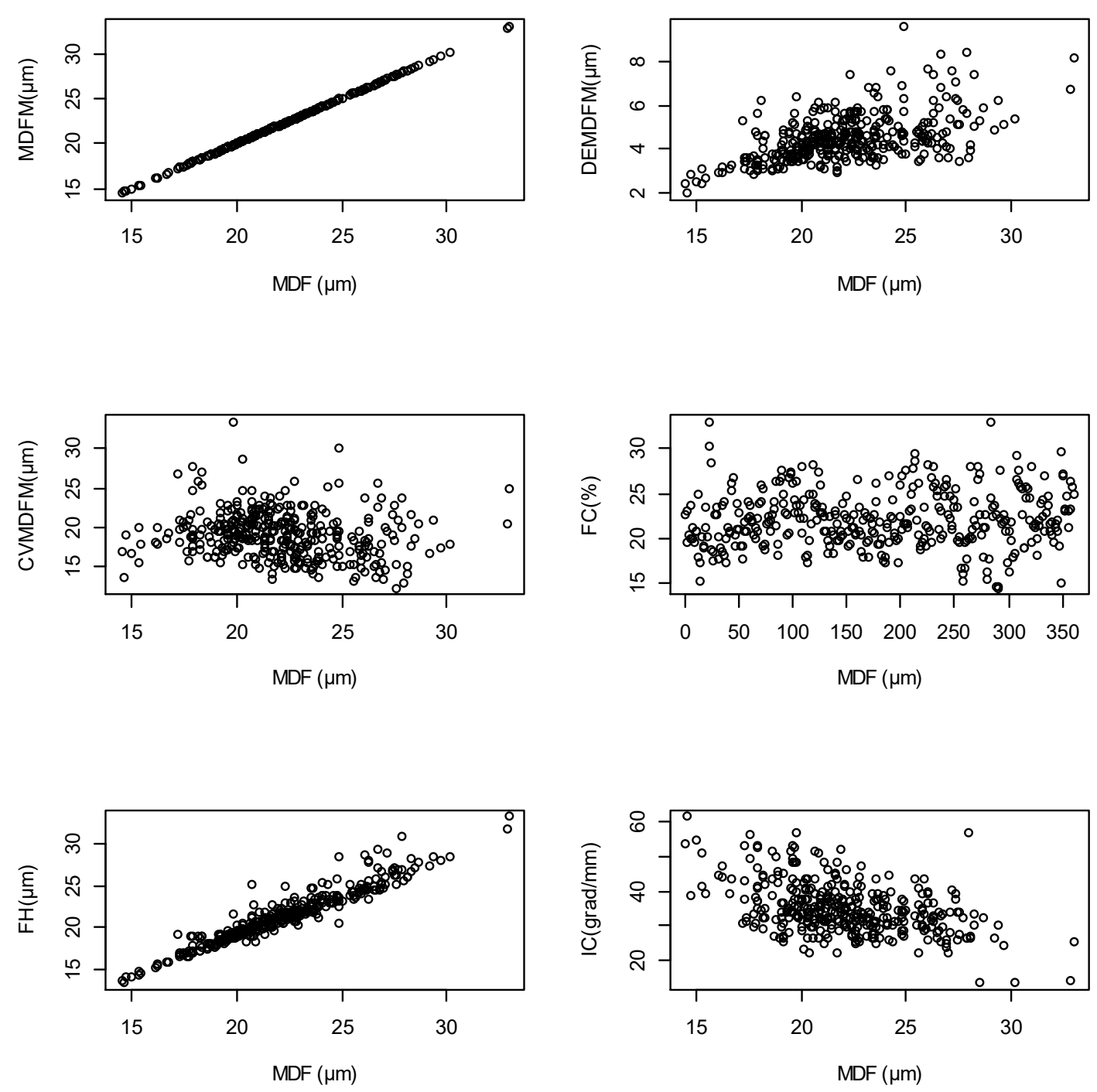

Figura 1. Correlaciones fenotípicas entre las características textiles de la fibra de alpaca del sector Queulla - Chapimarca, Grau, Apurímac

\section{Conclusiones}

- La fibra producida por las alpacas del sector de Queulla, comunidad de Chapimarca, distrito, Chuquibambilla, de provincia de Grau, Región Apurímac tiene buena calidad por sus cualidades de finura, factor de confort, finura al hilado y regular curvatura, y de este modo cum- ple con los requisitos que exige la industria textil nacional e internacional.

- Los machos presentaron un mejor FC y finura al filado (SF) que las hembras $(\mathrm{p}<0.05)$.

- Los animales más jóvenes tienen fibra de mejor calidad que la de los animales adultos; así mismo la fibra del costillar y de la grupa es de mejor calidad que la fibra de la paleta. 


\section{Literatura Citada}

1. Arizaca PF. 2018. Efecto de la zona de muestreo corporal y sexo en características textiles de la fibra de alpacas Huacaya del CIP La Raya. Tesis de Médico Veterinario y Zootecnista. Puno, Perú: Univ. Nacional del Altiplano. 85 p.

2. Barrionuevo E. 2019. Caracterización física de la fibra de alpaca Huacaya utilizando OFDA 2000 en cuatro comunidades de Ocongate-Quispicanchis. Tesis de Ingeniero Zootecnista. Cusco, Perú: Univ. Nacional San Antonio de Abad del Cusco. 74 p.

3. Baxter P, Cottle D. 2010. Fiber diameter distribution characteristics of midside (fleece) samples and their use in sheep breeding. International Wool Organisation Technical Committee Meeting. Report N. ${ }^{\circ} 12.8 \mathrm{p}$.

4. Botha AF, Hunter L. 2010. The measurement of wool fibre properties and their effect on worsted processing performance and product quality. Part 1: The objective measurement of wool fibre properties. Textile Progress 42: 227-339. doi: 10.1080/00405167.2010.486932

5. Bustinza V. 2001. La alpaca, conocimiento del gran potencial andino. Puno, Perú: Univ. Nacional del Altiplano. 495 p.

6. Bustinza V, Medina G, Fernández E. 1985. Crecimiento de la alpaca. En: V Convención Internacional sobre Camélidos Sudamericanos. Cuzco, Perú.

7. Castillo R, Zacarías A. 2014. Determinación de las características tecnológicas de los diferentes componentes del vellón de la alpaca (Vicugna pacos) Huacaya. Tesis de Ingeniero Zootecnista. Huancavelica, Perú: Univ. Nacional de Huancavelica. $130 \mathrm{p}$.

8. CENAGRO. 2012. IV Censo Nacional Agropecuario. Lima Ministerio de Agricultura. [Internet]. Disponible en: https:/ /www.inei.gob.pe/estadisticas/censos/
9. De los Ríos E. 2006. Producción textil de fibras de camélidos sudamericanos en el área altoandina de Bolivia, Ecuador y Perú. Viena, Austria: UNIDO. 52 p.

10. Fish VE, Mahar TJ, Crook BJ. 1999. Fibre curvature morphometry and measurement. Wool Tech Sheep Bree 47: 248-265.

11. Flores $W .2017$. Perfil de fibra, índice de confort e índice de curvatura en alpacas Huacaya del distrito de Corani Carabaya, Tesis de Médico Veterinario y Zootecnista. Tacna, Perú: Univ. Nacional Jorge Basadre. $101 \mathrm{p}$.

12. Franco F., San Martín F, Ara M., Olazabal J. y Carcelén F C 2009. Efecto del nivel alimenticio sobre el Rendimiento y calidad de fibra en Alpacas Rev Inv Vet Perú; 20 (2): 187-195.

13. Franco F, San Martín F, Ara M, Olazabal J, Carcelén F. 2009. Efecto del nivel alimenticio sobre el rendimiento y calidad de fibra en Alpacas. Rev Inv Vet Perú 20: 187-195. doi: 10.15381/ rivep.v20i2.605

14. Gil R. 2017. Evaluación de las características textiles de la fibra de alpacas Huacaya del Instituto de Investigación y Promoción de Camélidos Sudamericanos, Puno. Tesis de Médico Veterinario y Zootecnista. Puno, Perú: Univ. Nacional del Altiplano. $67 \mathrm{p}$.

15. IWTO-47. International Wool Textile Organisation. 2007. Measurement of the mean and distribution of fibre diameter of wool using an optical fibre diameter analyser (OFDA). In: IWTO Red Book Specifications. Brussels: IWTO.

16. Llactahuamani I, Ampuero E, Cahuana E, Cucho H. 2020. Calidad de la fibra de alpacas Huacaya y Suri del plantel de reproductores de Ocongate, Cusco, Perú. Rev Inv Vet Perú 31: e17851. doi: 10.15381/rivep.v31i2.17851

17. Lupton CJ, McColl A, Stobart RH. 2006. Fiber characteristics of the Huacaya alpaca. Small Ruminant Res 
64: 211-224. doi: 10.1016/j.smallrumres.2005.04.023

18. Machaca V, Bustinza A, Corredor F, Paucara V, Quispe E, Machaca R. 2017. Características de la fibra de alpaca Huacaya de Cotaruse, Apurímac, Perú. Rev Inv Vet Perú 28: 843-851 doi: 10.15381/rivep.v28i4.13889.

19. Mamani H. 2012. Principales parámetros físicos de la fibra a la categorización y clasificación de vellones de alpacas Huacaya blanca. Tesis de Médico Veterinario y Zootecnista. Puno, Perú: Univ. Nacional del Altiplano. $60 \mathrm{p}$.

20. Martínez C. 1999. Estadística y muestreo. Colombia; ECOE Ediciones. $886 \mathrm{p}$

21. Marler J, Hansford K, McLachlan I. 2002. A comparison of the performance of on-farm OFDA2000 and in-store FLEECESCAN - Part 1: The precision of the measured fibre characteristics of a fleece, In: International Wool Textile Organisation Conference. Barcelona, Spain.

22. Matute G, Holgado K, Vásquez I. 2009. Cluster alpaquero en la Región Puno. Lima, Perú: Universidad ESAN. $157 \mathrm{p}$.

23. McColl A, Lupton C, Stobart B. 2006. Fiber characteristics of U.S. Huacaya alpaca. Small Ruminant Res 64: 211-224.

24. McGregor BA, Butler KL. 2004. Sources of variation in fibre diameter attributes of Australian alpacas and implications for fleece evaluation and animal selection. Aust J Agr Res 55: 433442. doi: 10.1071/AR03073

25. McGregor BA. 2006. Production, attributes and relative value of alpaca fleeces in southern Australia and implications for industry development. Small Ruminant Res 61: 93-111.

26. McGregor BA. 2014. Variation in the softness and fibre curvature of cashmere, alpaca, mohair and other rare animal fibres. J Textile Inst 105: 597-608. doi: $10.1080 / 00405000.2013 .828448$
27. Meza M. 2018. Caracterización física de alpacas de color de la raza Huacaya en el distrito de Totos, Provincia de Cangallo, Región Ayacucho a 4,438 msnm. Tesis de Médico Veterinario. Ayacucho, Perú: Univ. Nacional San Cristóbal de Huamanga. 55 p.

28. Ormachea E, Calsín B, Olarte $U$. 2015. Características textiles de la fibra en alpacas Huacaya del distrito de Corani. Rev Invest Altoandina 17: 5-10.

29. Qi K, Lupton CJ, Pfeiffer FA, Minikhiem DL. 1994. Evaluation of the optical fibre diameter analyser (OFDA) for measuring fiber diameter parameters of sheep and goats. J Anim Sci 72: 16751679. doi: $10.2527 / 1994.7271675 x$

30. Quispe EC, Alfonso L, Flores A, Guillén H, Ramos Y. 2009a. Bases para un programa de mejora de alpacas en la región Altoandina de Huancavelica - Perú. Arch Zootec 58: 705-716.

31. Quispe EC, Rodríguez T, Iñíguez L, Mueller JP. 2009b. Producción de fibra de alpaca, llama, vicuña y guanaco en Sudamérica. Anim Genet Resour 45: 1-14. doi: 10.1017/S1014233909990277

32. Quispe JE, Apaza E, Quispe DM, Morocco N. 2016. De vuelta a la alpaca: la producción primaria en una perspectiva empresarial y competitiva. Puno, Perú: Ed Meru Corporación. 440 p.

33. Quispe JE. 2019. Efectos ambientales sobre el peso al nacimiento e incremento corporal al destete en alpacas del CIP Quimsachata, INIA- Puno. Rev Invest Escuela Posgrado UNA 8: 931-943. doi: 10.26788/riepg.2019.1.114

34. Quispe JE, Apaza E, Olarte C. 2021. Características fisicas y perfil de diámetro de fibra de alpacas Huacaya del Centro Experimental La Raya (Puno, Perú), según edad y sexo. Rev Inv Vet Perú 32: e20004. doi: 10.15381/rivep.v32i2.-20004

35. Roque L, Ormachea E. 2018. Características productivas y textiles de la fibra en alpacas Huacaya de Puno. Perú Rev Inv Vet Perú 29: 1325-1334. doi: 10.15381/rivep.v19i4.14117 
36. Ramos V. 2018. Características fenotípicas de la fibra de alpaca Huacaya en la región Apurímac. Tesis de Segunda Especialidad. Puno, Perú: Univ. Nacional del Altiplano. $87 \mathrm{p}$.

37. Renieri C, Pacheco C, Valbonesi A, Frank E, Antonini M. 2007. Programa de mejoramiento genético en camélidos. Arch Latinoam Prod Anim 15: 205-210.

38. Vázquez R, Gómez O, Quispe E. 2015. Características tecnológicas de la fibra blanca de alpaca huacaya en la zona altoandina de Apurímac. Rev Inv Vet Perú 26: 213-222. doi: 10.15381/ rivep.v26i2.11020
39. Wang X, Wang L, Liu X. 2002. A study of Australian alpaca fibres. In Proc Third China International wool textile conference. China.

40. Wood E. 2003. Textile properties of wool and other fibers. Wool Tech. Sheep Breed. 450 p.

41. Wuliji T, Davis GH, Dodds KG, Turner PR, Andrews RN, Bruce GD. 2000.

Production performance, repeatability and heritability estimates for live weight, fleece weight and fiber characteristics of alpacas in New Zealand. Small Ruminant Res 37: 189-201. doi: 10.1016/ s0921-4488(00)00127-9 